\title{
Dynamics in the practice of structural design: the problems of implementation
}

\author{
O. S. Saar \\ Sircovich Saar Civil Engineering, Jerusalem, Israel
}

\begin{abstract}
This paper stresses the difficulties practising engineers are facing in order to keep pace with the continuously evolving knowledge on structural dynamics, and suggests possible ways to make this knowledge accessible to them towards implementation in the practice of structural design.

Keywords: problems of implementation, practising engineer, design situation for statics or dynamics, designers approach, online journal.
\end{abstract}

\section{Introduction}

It is very well known that a remarkably great number of engineers in the practice of structural design, all over, are confronted with difficulties when dealing with a dynamic design situation. This paper is intended to focus attention on this anomaly and to outline some possible implications.

\section{The problems of implementation}

Structural dynamics is, no doubt, the most complex subject that underlines the broad knowledge required from an engineer in order to perform the practice of structural design. Because of that, engineers are trying continuously to enlarge their professional knowledge by looking for sources of additional learning on the issue, such as technical books, journals, publications, descriptions of particular dynamic events or any information that can enrich their understanding or provide them with guiding tools for practical application. But, going through those sources reveals itself to be a very hard, sometimes impossible task, since sooner or later they are faced, unfortunately, with familiar concepts that had already lost a clear quantitative meaning such as damping, hysteresis, impulse, or with 
mathematical elaborations that are difficult to follow, since they are not a part of their daily occupation.

For a vast number of engineers in design offices, dynamic design situations that depart from the Codes or are not included there at all, are sporadic events, as opposed to static design situations, which comprise the bulk of their work.

The main difference between a static and a dynamic design situation, for a structural engineer, can be described through a comparison between two design situations of a simple, statically determinate beam in the elastic stage, namely when under a static compared to a dynamic load; in the static design situation, calculations and design can be performed directly. By contrast, in the dynamic design situation, the external load, which varies with time, imparts on the structure dynamic forces those themselves vary with time; an interdependence is therefore created between the external load and the geometrical-mechanical characteristics of the beam and calculations and design become more complicated than those typical of a static design situation. In some cases, calculations can reach highly sophisticated levels, requiring a special mathematical training that is uncommon in the practice of structural design.

In the present computer age it is quite normal for the engineer to rely on the correct use of computers for a solution that satisfies the requirements of safety and performance. Practising engineers should not have to deal with the mathematical formulation and solution of the differential equations of equilibrium. In order to deal with problems of structural design they should have mainly a clear and thorough understanding of the dynamic issue.

Mathematical treatment of a dynamic case is indeed the right way to find the response of a structure in a particular dynamic design situation; this makes the issue closer to the activity of an expert than to that of an engineer facing a practical problem to solve.

Because of that a clear distinction should be made, when considering dynamics in structural design, between those regarding academic activities and those to be applied by the engineer in the daily practice. In the following table a comparison is made of a similar work performed by a structural engineer and by an academic:

\section{$\underline{\text { Structural Designer }}$}

1. Geometrical-mechanical definition of the structure.

2. Input of structure's model and its loads into a computer program.

3. Interpretation of graphic and numerical output as required for the design of the structure.

\section{Expert's Approach}

1. Physical and mathematical modeling; formulation of the dynamic equation of motion.

2. Mathematical solution of the equation of motion.

3. Formulation of structure's response function; elaboration of response curves. 


\section{The practising engineer}

Practising structural engineers develop with time a certain 'feel' for structures in their daily work, which is actually the result of an extensive practice together with a deep and correct professional knowledge. To develop such an "intuition" for a dynamic design situation, the engineer should pay special attention to other factors, in addition to the stiffness $k$, which is characteristic of a static design. These are the damping ratio $\xi$, and mass $\mathbf{M}$; but since those three factors are interacting, the engineer finds it very hard to trail along a trial and error path.

The engineer tries usually to focus his/her attention, whenever possible, on the physical meanings of concepts related to dynamics: the period " $T$ " and the natural frequency " $f=1 / T$ " for example, have very clear physical meaning, but then arises the question what is the physical meaning of " $\Omega$ ", the natural circular frequency of a structure? Does it represent, by chance, the circular rotating motion of a mass in a rotating machine? If so, how is it connected, for example, with the movement of a mass in a reciprocating machine? He can try perhaps to give a physical explanation saying that $\Omega$ measures indeed the vibrating movement of the structure as a motion along a mathematical " $2 \pi$ " circle. In this manner $\Omega$ will represent the number of hypothetical circles per second.

For academia the natural circular frequency belongs to the beautiful construction of the world of mathematics that makes possible to develop and achieve the present and continuously evolving knowledge in structural dynamics; in practice, " $\Omega=2 \pi f$ " is measured in radians per seconds.

The world of design of the structural engineer is full of questions when facing a dynamic situation which is not a regular structure subjected to earthquake or wind loads; that design situation is covered within the Norms; but, how should he/she proceed if for example the span of a bridge is in the transition category limits, or when designing a building with irregular plan, or when some construction details correctly designed in his office were changed, unintentionally, at the construction site, or when he/she would like to ameliorate the response of a structure by introducing limited changes in the foundation?

Actual codes do not always provide an adequate treatment to a variety of dynamic events that may require consideration in the process of design, such as external dynamic loads produced by human activity (dancing, running, skipping, etc.), operating machinery (turbine, printing press, forging, etc.), road/rail traffic, construction activities (piling, blast excavation, heavy compaction, etc.), collisions of cars or airplanes, collapse of ground foundations, etc.

Proper consideration of dynamic effects on structures such as overstressing, vibrations and fatigue of structural materials is becoming more important with the modern tendency to design slender and elegant structures. Those structures are the result of conscious professional efforts to stress the engineer's capabilities, the use of stronger materials, and the implementation of sophisticated building technologies. 
Among the consequences that the engineer should be aware of are not only building damages, but also physiological and psychological effects on people, degradation of machine performances, and more. These effects cannot always be quantified in the output of computer calculations, but the engineer is expected to take adequate preventive measures whenever possible.

Unfortunately, for some of these effects there are neither satisfactory answers in the Codes nor sound and strong applicable rules or reliable guidelines. True, there are dozens of periodic publications on dynamics, but none of these again is oriented to the daily practice of structural design; this creates an intolerable situation for the practising engineer who can not find with relative ease, the answers he/she is looking for.

\section{The practice of structural design}

The need for easy answers is pertinent to the practice of structural design: the latter is a highly absorbent and intensive daily work, with a variety of dominant activities, besides the proper dedication to statical calculations and design.

A description of the practice of structural design would include first:

- a preliminary technical phase of coordination with other professionals of the design team, consultations with contractors and budget considerations.

- a creative phase of conceptual elaboration of a structural solution, including structural materials, basic dimensions and site construction considerations.

- a phase of preliminary statical calculations, based on already accorded geometry .

- a phase of preliminary appreciation of costs including discussions with owner and architect.

Only then does the right time arrive for comprehensive statical calculations and preparation of construction drawings and detailing, followed by computations and special remarks for tender.

This varied and intensive list of activities, performed during a long period of time, is still to be complemented with top site construction supervision which will include, most probably, corrections of construction errors, which are not so easy to elaborate.

\section{Didactic tools}

Along the last fifty years, authors expressed their concern about the difficulties to make structural dynamics accessible, both to students and engineers in the daily practice.

"students find the mathematical manipulation so intriguing that they fail to develop the physical understanding essential for good design"; Biggs [1].

"In developing this book, much emphasis has been placed on making structural dynamics accessible to students and professional engineers because many find this subject to be difficult"; Chopra [2]. 
More recently the book "Dynamics in the Practice of Structural Design" [3] came about as a result of the author's close awareness, as a professional, of the world of the practising engineer. It is therefore emphatically user-friendly.

Since structural dynamics is a subject of evolving knowledge, practising engineers understandably need continuous updating. They are conscious of this need and avidly seek such knowledge. Hence it is very important that information/learning material be made accessible to them.

Conferences like ERES are very important, rich and highly instructive in the topics they embrace. They should be complemented with other didactic tools that will be then made available to practising engineers, wherever they are, on a periodic basis.

The academic community should stand behind this platform since the multifacet work of the practising engineer and the enormous responsibility he carries on his shoulders, entitles him to expect their support.

\section{References}

[1] An Introduction to Structural Dynamics. John M. Biggs 1964. McGraw-Hill, Inc.

[2] Dynamics of Structures. Anil K. Chopra 1995. Prentice Hall

[3] Dynamics in the Practice of Structural Design. Oscar Sircovich Saar 2006. WIT Press 